\title{
Optimising Self-Similarity Network Traffic for Better Performance
}

${ }^{1}$ Ikharo A. B., ${ }^{2}$ Anyachebelu K. T., ${ }^{3}$ Blamah N. V., ${ }^{4}$ Abanihi V. K.

${ }^{1,4}$ Department of Electrical \& Computer Engineering, Edo University Iyamho, Nigeria

${ }^{2}$ Department of Computer Science, Nasarawa State University, Nigeria

${ }^{3}$ Department of Computer Science, University of Jos, Nigeria

\section{Article Info}

Volume 7, Issue 4

Page Number: 164-176

Publication Issue :

July-August-2020

\section{Article History}

Accepted : 05 Aug 2020

Published : 12 Aug 2020

\section{ABSTRACT}

Given the ubiquity of the burstiness present across many networking facilities and services, predicting and managing self-similar traffic has become a key issue owing to new complexities associated with self-similarity which makes difficult the achievement of high network performance and quality of service (QoS). In this study ANN model was used to model and simulate FCE Okene computer network traffic. The ANN is a 2-39-1 Feed Forward Backpropagation network implemented to predict the bursty nature of network traffic. Wireshark tools that measure and capture packets of network traffic was deployed. Moreover, variance-time method is a log-log scale plot, representing variance versus a non-overlapping block of size $\mathrm{m}$ aggregate variance level engaged to established conformity of the ANN approach to self-similarity characteristic of the network traffic. The predicted series were then compared with the corresponding real traffic series. Suitable performance measurements used were the Means Square Error (MSE) and the Regression Coefficient. Our results showed that burstiness is present in the network across many time scales. The study also established the characteristic property of a long-range dependence (LRD). The work recommended that network traffic observation should be longer thereby enabling larger volume of traffic to be capture for better accuracy of traffic modelling and prediction.

Keywords : Bursty, Backpropagation, Traffic, Network, Self-similarity, Prediction

\section{INTRODUCTION}

A general view of computer networks show one of continuous growth in the number of hosts, capacity of infrastructure, variety of applications and other associated devices. Therefore, a scalable architecture for service differentiation must be able to accommodate this continued growth [4]. Recent studies have shown that conventional traffic models have been found not to capture real situation in network infrastructure rather that self-similarity is a better model in representing traffic pattern [2]. Modelling and prediction are two factors that must be adopted to assist network administrators predict 
network behaviours, work out remediation for better informed decision.

Traffic prediction is one of the most important tasks in traffic management because an accurate prediction is able to help in improving network performance [2]. Analysis of traffic in deployed computer networks is important for determining performance, reliability and scalability. The volume of traffic on the network has become as issue that demand attention and this is complemented with traffic congestion, transmission delays and outages, packet loses and cross link problems. For these reasons time series models are introduced in network traffic simulation and prediction [15].

According to [11], network traffic predictability is of important benefits in many areas, such as dynamic bandwidth allocation, network security, network planning and predictive congestion control. This can be used to improve Quality of Service (QoS) mechanisms as well as for optimal resource management.

According to [13], self-similar object is exactly similar to a part of itself. Various techniques are being used by researchers for network traffic analysis [10]. Self-similarity has pronounced impacts on the network performance. However, self-similar model is based on fractal theory, and can be described using the Hurst parameter and Long-Range Dependence (LRD) [3]. The Hurst parameter represents the measurement of self-similarity and variability of packet arrival rate. There are several different methods for estimating the Hurst parameter which can lead to diverse results [3].

Neural Network exhibits degree of self-similarity at large scale and high degree of multi-fractal at small time scale. Network traffic condition is a very complex system and nonlinear in nature, therefore, Artificial Neural Network (ANN) is highly suitable for the situations where the underlying processes exhibit chaotic features. In this work, pattern recognition algorithm such as ANN will be used in order to predict the presence of traffic congestion because studies have proved its accuracy.

Federal College of Education (FCE), Okene campus network is bursty in nature and many data packets are lost and often time data packets sent never get to their destinations. Also, the network is very slow, experiencing frequent outages and losses in connections. The aim of this work is to provide optimise network traffic performance for FCE Okene network system using Artificial Neural Network (ANN) model in MATLAB software.

\section{LITERATURE REVIEW}

A number of researchers have addressed network traffic prediction methods by various means using various techniques and applications. Several of these well-known prediction models have met a few crucial problems. Some studies abound that used ANN in order to predict time series. Others used ANN for financial time forecasting [12] and that ANN could understand self-similarity characteristic and long range dependency among data packet [13]. Self-similar traffic model has been proposed to be a more suitable model in representing high-speed traffic [2]. [22] presented definitions of self-similarity and LDR, focusing on their physical interpretation, and highlights the relevance of these properties in network dimensioning. [23] employed the application of adequate mathematical models of traffic to correctly assess the characteristics of the quality of service (QoS) of the network.

[12] worked on an adaptive network traffic prediction algorithm based on Back Propagation (BP) neural network. [10] worked on a time-series prediction model for the Packet Loss Rate (PLR). [1] worked on the analysis of the network traffic over IP 
network by developing ANN model using multi-layer perceptron. [21] presented a feasibility study on the effect of influence of traffic type on performance of routing algorithm while the routing algorithm is treated as one of the mechanisms to support QoS in the network.

[19] used wavelet analysis and Hopfield neural network for network traffic forecast. This formed the basis for his research on network traffic prediction model. [18] worked on the analysis that the noninterference of network traffic leads to network traffic congestion and paralysis. [14] worked on the development of ANN methodology for network resource management and network traffic congestion control. From the findings, ANN can better handle non- linear data relationship between the input and output patterns. And that the model is able to improve the prediction precision, and has the good flexibility to the network management.

\section{MATERIALS AND METHOD}

\subsection{Data Source and Data Collection}

Collection of network data set from the FCE Campus Network is made by using Wireshark sniffer to provide captured packets of network traffic with information on packet size, type and Transmission Control Protocol (TCP) connections. A quantum number of packet sizes captured over 2 hours would suffice for this study. Data used for the study involved a five distinct nonsuccessive days. However, only the readings with the highest number of records were used for the purpose of this research to minimise statistical errors.

\subsection{Modelling with Variant-Time Plots}

According to [26] based on the definition of selfsimilarity, we can say that the aggregated processes, $\{\mathrm{Xk}(\mathrm{m})\}$ are strictly self-similar if,

$$
\operatorname{var}\left(X^{m}\right)=\sigma^{2} m^{-\beta}---(1)
$$

with a Hurst parameter, $H=1-\beta / 2$. So this indicates that if we form the aggregated processes, $\{X(m)\}$ for various values of $\mathrm{m}$, and plot the logarithms of variances against the logarithms of $\mathrm{m}$, then it will yield a straight line, the slope of which will give us the value of $\beta$.

\subsection{Modelling and Simulation with ANN}

Using graphical user interface (GUI) of the neural network model and the idea of [24], an N-S-1 feed forward backpropagation network was employed to predict the traffic behaviour of the network. For the prediction of the self-similarity network, a 2-39-1 feed forward backpropagation network ANN was implemented, which comprised of the input layer, a linear output layer, and a tangential-sigmoid hidden layer. The selection of the tangential-sigmoid transfer function for the hidden layer allows any nonlinear relation between the system predictors and the output. A hard-limit transfer function for the output layer was used for the direct prediction of burst presence through the hard-labels of the output. In this case, even if a direct result can be obtained, the models output cannot be adjusted according to a desired level of sensitivity and specificity threshold. This is achieved by training the network with the sample data until optimal value that best maximize the model performance is achieved. The number of neurons used in the hidden layer was chosen based on [17] method and corresponds to the value that also maximizes the model's performance. The selected criteria for ANN model is $\left(4 n^{2}+3\right) /\left(n^{2}-8\right)$ using 39 numbers of hidden neurons and obtained a minimal MSE value [17]. The network predictors as shown in Figure 1 consist of a 2 x 2561 matrix, where each row represents the packet size and packet count respectively for a total of one hour per dataset, while each column stands for the total number of data used. The target vector has a binary form; its elements being 1 in case of burst 
presence for the specific packet data and $\mathbf{0}$ in case of burst absence.

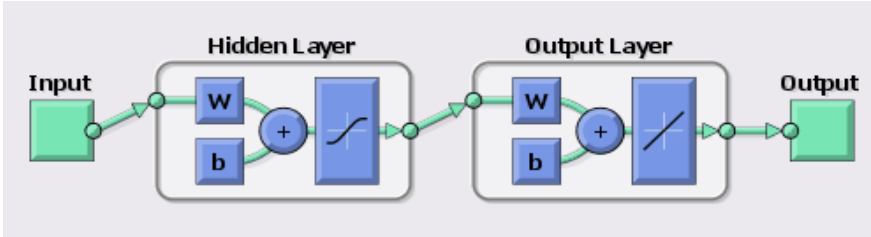

Figure 1: Artificial Neural Network Architecture

\section{RESULDS AND DISCUSSION}

Captured packets of two (2) different network traffics (FCE network and standard MTN network) using Wireshark Sniffer were made in real-time. These measured traffics were used for the study analysis. The captured traffic outcomes are shown in Figure 2, FCE DataCap8 depicts instance of exhibited layout at various time scales. Figure 2 (a) is at time scale of 1 second. The profile captures all packet data and TCP error. It simply indicates the number of packets per unit second between transmitted and received. Figure 2 (b) is at time scale of 10 seconds and Figure 2 (c) is at time scale 1 minute. Figure $3(\mathrm{a})$ to $(\mathrm{c})$ is for DataCapMTN and are also of the same time scale as Figure 2.

These figures depicted sequences of plots of the data packet counts, that is, a number of packets per unit time for three different traffics. Starting with a time unit of zero second with subinterval of 100 seconds per slot. A surface observation of these profiles indicate that figure Figure 2 shows cyclic pattern with peak values of data packets at 6 other points in the time axis. Figure 3 shows a saw-tooth like pattern with three peak values between 600 and 800 seconds, 1500 and 1800 , and between 2500 and 3000 seconds. In a general sense, the three profiles are self-similar in the same time scale - bursty in their characteristics. In particular, notice must be drawn to the fact that there is the absence of a natural length of a burst, but simply consist of bursty subperiods separated by less bursty subperiods. These patterns exhibited by these profiles suggest the use of self-similar stochastic processes for their traffic modellings. As a result of this, subsequent sections is devoted to give detailed explanation and situation of the effects obtained after deploying our models to analyse the self-similar stochastic process.

The increase of packet count distribution of Figures 2 and 3 is due to aggregation. Apart from the scaling property, the figures appear to share identical distribution characteristics. In other words, if the tenfold multiplication of packet count dimensions (incurred at each aggregation step) were to be compensated by division, the sequences would be indistinguishable. That suggests the plots have similar statistical properties.

One major feature that is prominenet and dominant throughout all the shown time-scales is burstiness. Bursts occur when traffic intensity peaks to around the average traffic level, and are clearly observed at all time-scales in the entire Figures. Furthermore, bursts have no natural duration; they appear at all timescales and themselves consist of higher-time-resolution bursts.

\subsection{Network Traffic Traces}

In our study we analysed two traffic traces DataCap8 for FCE network and DataCapMTN for Mobile Telephone Network (MTN) network as outlined in table 1 below.

\section{Table 1: Summary of Traces Used in the Study}

\begin{tabular}{lll}
\hline Name & Observation & Duration \\
\hline DataCap8 & 65963 & 35 Min \\
DataCapMTN & 174205 & 1 Hour \\
\hline
\end{tabular}

In order to analyse the self-similarity of these traces we have calculated the Hurst parameter with the 
help of [9]. Excel worksheet was developed to ease the estimation of Hurst parameter computation. The results had enabled the profiles of Figures 4 and 5 . The variance-time curve shows an asymptotic slope that is easily estimated to be approximately 0.81 for DataCap8 and 0.93 for DataCapMTN. These outcomes enabled the assessment of the Hurst parameter $H$ for the traffic traces. These $H$ values will help us ascertain their placement in the standard gauge in terms of acceptability, relevance and interpretations in the scheme of traffic analysis. The obtained values fall within the brackets of $0.5<\mathrm{H}<1$. And the $H$ being a measure of the level of self-similarity of a time series that exhibits long-range dependence and short-range dependence. Therefore, DataCap8 and DataCapMTN both exhibited self-similarity with Long Range Dependence (LRD).

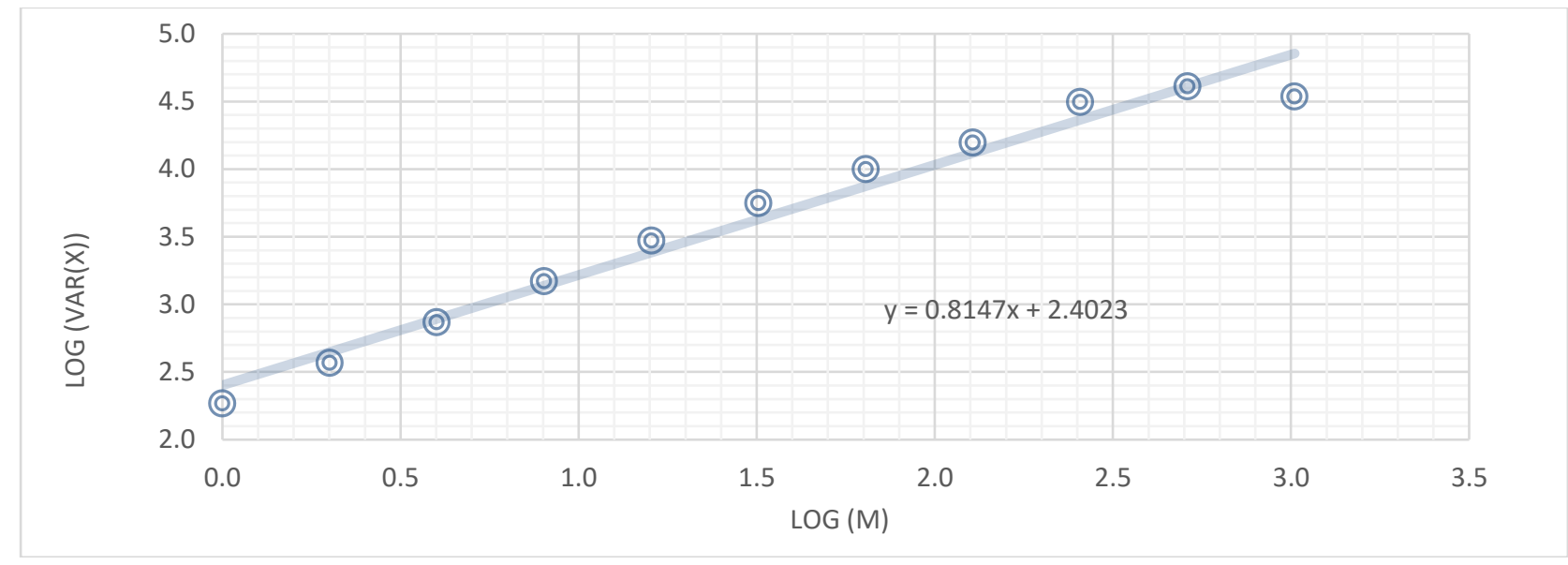

Figure 4 : Hurst Parameter Estimation with Variance-Time Plot for DataCap8

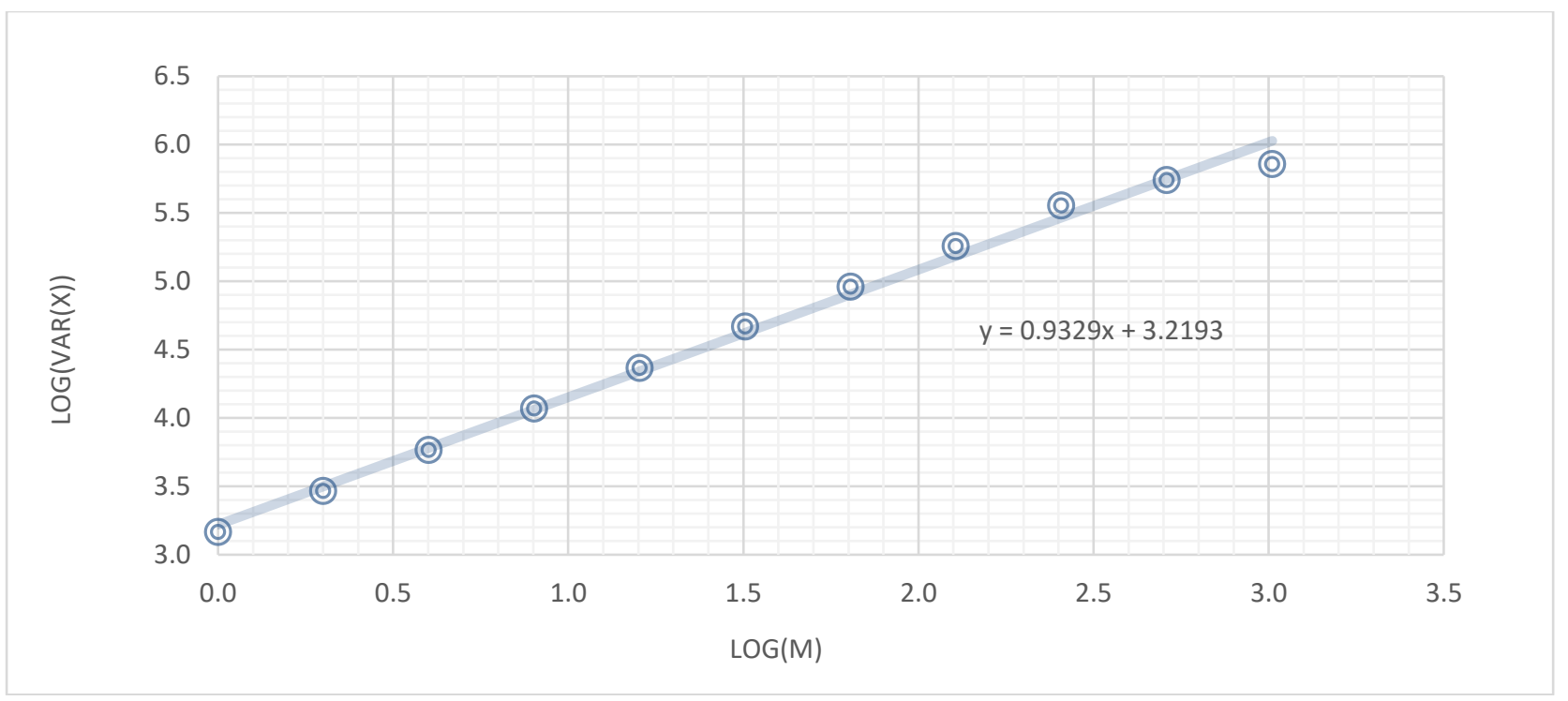

Figure 5 : Hurst Parameter Estimation with Variance-Time Plot for DataCapMTN 
In the case of FCE DataCap8 and DataCapMTN traffic, the autocorrelation function decayed hyperbolically, which means, that these traffics have long-range dependence characteristics.

\subsection{Implications of the Variant-Time Model on the Hurst Parameter}

The Hurst parameter $H$, as a measure of the extent of long-range dependence in a time series, takes on values from 0 to 1 . A value of 0.5 indicates the absence of long-range dependence. The closer $H$ is to 1 , the greater the degree of persistence or long-range dependence. $H$ less than 0.5 corresponds to less persistence otherwise known as Short-Range Dependence (SRD), which indicates strong negative correlation so that the process fluctuates unpredictably.

By estimating the Hurst parameter $H$, we have simply determine the attributes of the traffic characteristics by obtaining values that indicate the long-time memory of the network traffic time series. It also shows that the time series of the network traffic is self-similar. A variant-time analysis in this case has been used on the given dataset to estimate the Hurst parameter. The results obtained showed agreement with results stated in literatures. Since DataCap8 and DataCapMTN traffic trace exhibited LRD, one way of describing the situation of their stationary process is in terms of autocovariance functions. For a long-range dependent process, the coupling between values at different times increases strongly as the time difference increases. The autocovariance does not drop to zero after a certain time-lag, and has a hyperbolic decay.

\subsection{ANN Model Implementation and Evaluation}

In a bid to ensure that ANN predicted traffic burst rate are in conformity with the desired outcomes, comparison of the measured traffic results is as shown in Figures 6 and 7. The two traffic traces were simply selected for the duration of the captured data and the outcome plotted using clustered column graphs to illustrate the comparison. Figure 6 shows an overlap of the column line graph for both measured and predicted values of DataCap8 of the same FCE network at different day and time. This is an indication that the ANN results showed conformity with desired burst rate output.

Also, a column chart plots for DataCapMTN, that is, Figure 7, for MTN network shows the same trend as indicated in DataCap8 of FCE Network profiles in Figure 6 previously. By extension, the ANN predictions for any of the network traffic for each location will behave similarly. Therefore, any inference based on these predictions could be said to be conclusive using ANN model and Variant-time plot.

Figure 6 also exhibited burst presence. The burst rate at the start of the traffic is 0.58 , which is the highest value in its entire duration of flow. The values significantly drop to much lower values as the distribution gets larger. It is observed that the burst rate becomes constant or steady as the traffic periods elongates.

Figure 7 displays marked presence of burst. The burst rate of 0.39 , which is the highest recorded value at the start of the traffic and it is significantly low, its distribution is also small. It is observed here that as the distribution expands or get larger, the burst rate is relative more consistent and having lower values of burst rates at higher distribution. 


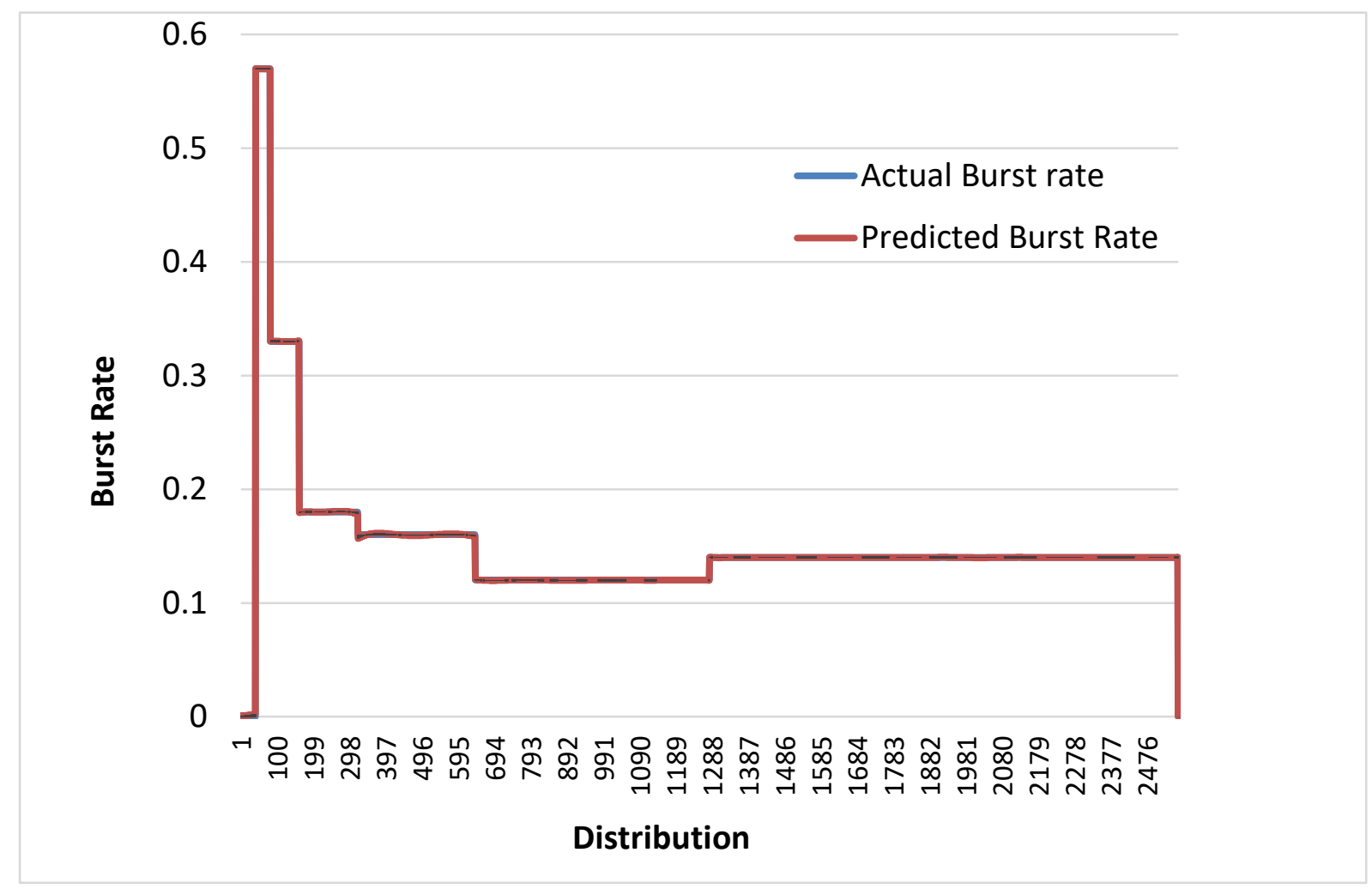

Figure 6: Profile of DataCap8 Measured and Predicted for FCE Network Traffic Compared

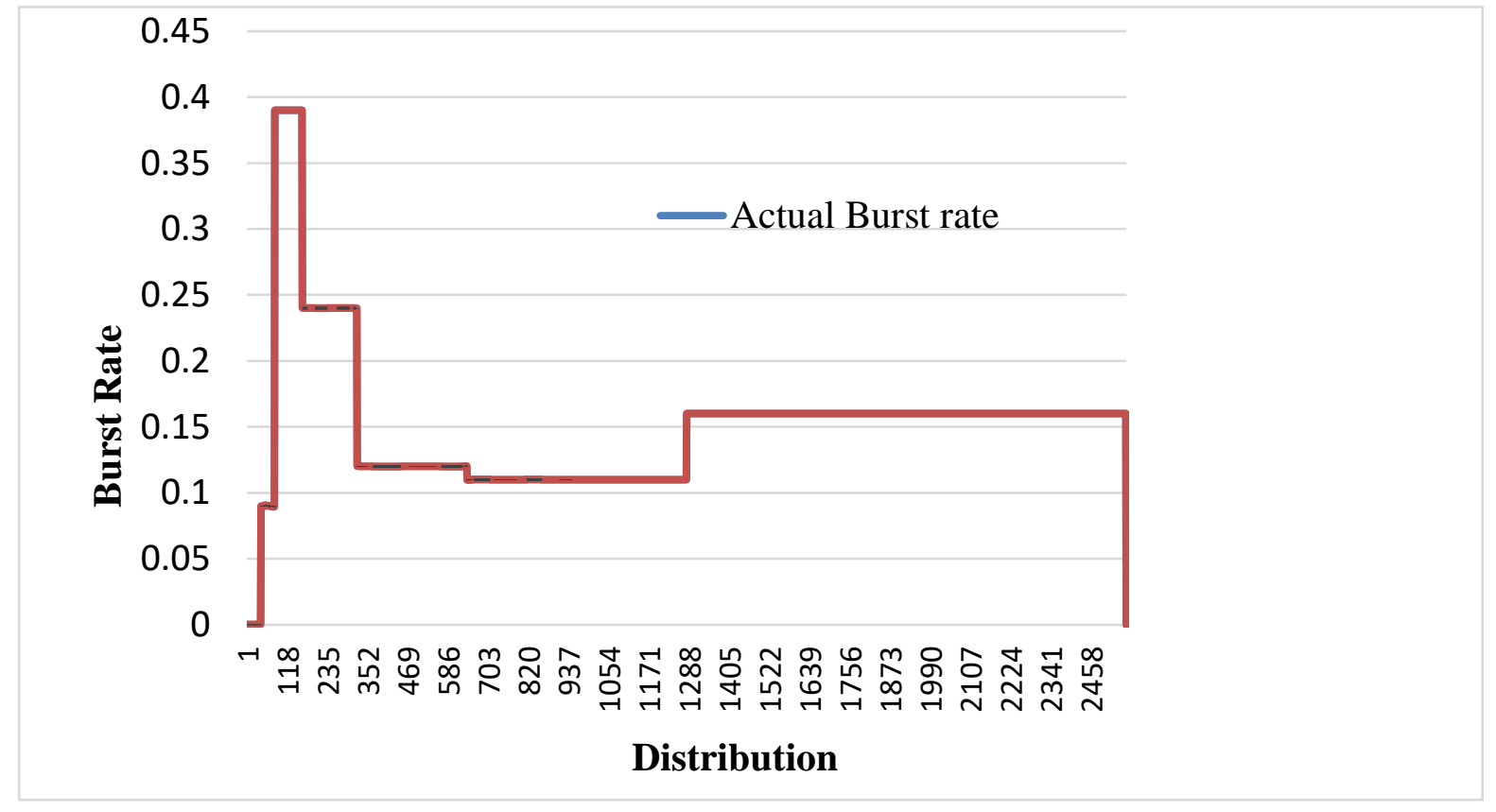

Figure 7: Profile of DataCapMTN2 Measured and Predicted for MTN Network Traffic Compared 
One common observation in Figures 6 and 7 is the fact that as the distribution expands or get larger, the burst rate is relative more constant and having lower values of burst rates. Interestingly, we noted that at the start of the traffic all profiles showed burst rates at their peak values, that is, hitting a maximum and then begin to decrease as the distribution gets larger with network traffic stabilisation. This initial high burst rates can be attributed to the system ability to exhibits one or all of these; the nodal degree of the network, burst arrival rate, load range of the network and reconstruction of the switch network during contention resolution as also validated by [25]. Packet drops and delays, possible disconnections and abrupt gains in network connections during normal system operations of FCE network is experienced. With the outcome of our ANN, the predicted data can be used to correctly analyse future FCE network behaviour and chat a better course or proffer solution to some of the challenges encountered by the FCE computer network.

\subsection{Evaluating ANN Network Performance}

The ANN model prediction performance indices are shown in Table 2. The various values for the epoch, mean square error (MSE), regression and net performances are given accordingly. The total number of data used in the ANN Network is 2561 in which $60 \%$ (1537) of these data is for the training phase with $20 \%$ (512) for validation and $20 \%$ (512) for testing. While it is found that the ANN model can make predictions with high degree of accuracy, the learning rate of the network is about 0.015 .

Figure 1 is the simulated ANN model that provided the platform on which Figures 8 and 9 were realised and the performance indices were obtained as indicated in Table 2 for the predictions.

Table 2: Performance Indices for the ANN Prediction

\begin{tabular}{ccccc}
\hline Locations & Epochs & MSE & Regression & Performances \\
\hline DataCap8 & 100 & $6.6956 \mathrm{e}^{-3}$ & 0.99 & $2.355506 \mathrm{e}^{-7}$ \\
DataCapMTN & 100 & $5.59213 \mathrm{e}^{-3}$ & 0.99 & $1.80957 \mathrm{e}^{-6}$ \\
\hline
\end{tabular}

Figure 8 represents a sample of the ANN training outputs for DataCap8 and DataCapMTN as they converge. According to [20] and [17] small training dataset (less than $5 \%$ ) provides high prediction error indicating insufficient dataset to adapt the ANN model parameter to the network characteristics. Thus subscribing to the use of higher percentage value of which $20 \%$ is an acceptable value. In thus study, we used 20\%, sufficient to improve our prediction accuracy or traffic predictability. Moreover, enlarging the training dataset does not really improve the prediction error because the model will be over trained. Our use of $20 \%$ for test are quite adequate and that ANN model has capture during training phase, the strong correlation of the traffic with Long-range dependence (LRD).

The performance displayed by figure 9 shows the rapid convergence of the training, validation, test output of the ANN network dataset to correctly predict the traffic scenarios. 
Figure 9 represents a sample of the regression analysis outputs and targets for these traffics.

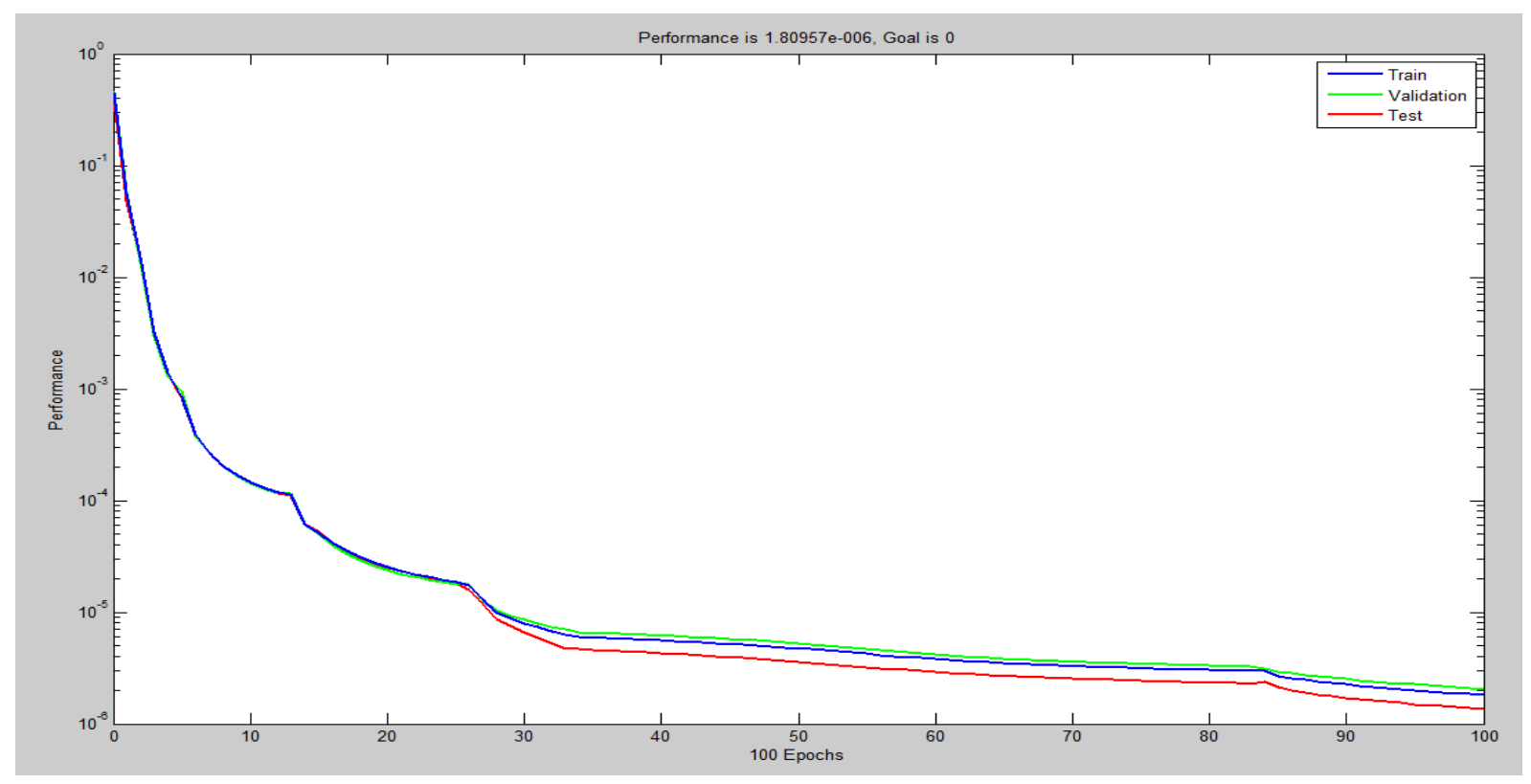

Figure 8: ANN Training Outcome for DataCapMTN

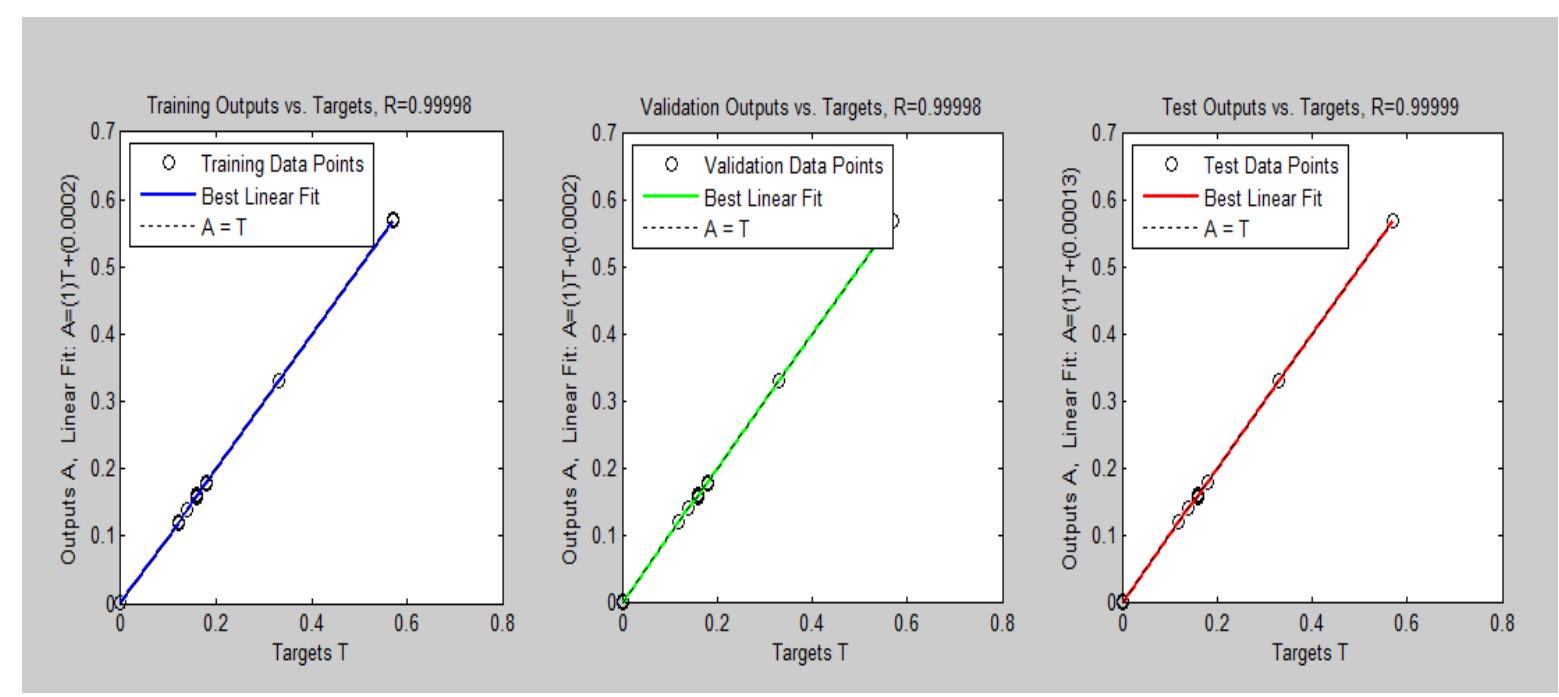

Figure 9: Regression Analysis of Outputs and Targets for DataCapMTN

\section{SUMMARY AND CONCLUSION}

Artificial neural networks (ANN) model and varianttime model were deployed to predict network traffic behaviour of FCE Okene computer network traffic.
These models were set in place to test the traffic network self-similarity behaviour and established the fact that the bursty nature of the networks are such as provide leverage for better performance and enhance quality of service. 
During the analysis phase we pay attention to the selfsimilar property and the Hurst parameter, which are the basic model for describing today's network traffic. In our FCE Okene network traffic analysis, we have established that network traffic (DataCap8 and DataCapMTN) can exist where Hurst parameter is bigger than 0.5 with the process having the property of a long-range dependence (LRD).

There was mild discrepancies between the captured and the simulated network traffics in the sense of packet-rate, bursts intensity, and variances. This could be attributed to the failure on the part of signal quality during data capture for analysis purpose. Even though, efforts were put in place to carefully eliminate some of these traffic errors. We cannot affirm that this is the best method for all situations, because of some embedded limitations, although it shows good results through simulation. It is important to point here that our study did not consider effect of data packet losses and the network load threshold predictability on the network traffic.

The study's results revealed that on the average, FCE traffic network performance improves with the increasing number of data packet distribution thereby providing a steady flow of burst over the entire period of system load. The studied network was found to be statistical self-similar which is to the level of selfsimilarity in the acceptable range of 0.5 to 1 . Also, that the network traffic has the characteristic property of a long-range dependence (LRD). The work recommended that network traffic observation should be longer to enable capturing larger volume of traffic for better precision of traffic modelling and prediction.

\section{REFERENCES}

[1]. S. Chabaa, A. Zeroual and J. Antari. "Identification and prediction of internet traffic using artificial neural networks.” J. Intelligent Learning Systems \& Applications, (2): 147-155, 2010.

[2]. C. O. Chow and K. A. Noordin. "Neural selfsimilarity traffic prediction in ATM networks." Georgian Electronic Scientific Journal:C Computer Science and Telecommunications, 1(5): 28 - 35, 2005.

[3]. M. Fras, J. Mohorko and Z. C. Učej. "Modeling of measured self-similar network traffic in OPNET simulation tool." Informacije, Ljubljana, 40(3): 224-231, 2010.

[4]. S. A. Himanshu, R. D. Smitta and B. Ramakanta. "The impact of self-similarity network traffic on quality of services (QoS) of telecommunication network." International Journal of IT, Engineering and Applied Sciences Research (IJIEASR), 2(2):54-60, 2013.

[5]. https://brilliant.org/profile/john-9143r5/about/

[6]. https://en.wikipedia.org/ wiki/Computersimulation

[7]. https://www.fd.cvut.cz/department /k611/pedagog/THO_A/A_soubory/stoch_proc1.pdf

[8]. https://www.techopedia.com/ definition/29917/networktraffic\#: :text=Network \%20traffic\%20

[9]. J. S. Huang. Accessed on 5 May 2020 from https://www.research gate.net/publication/272792633 Excel_Hurst_Calculator /link/54eea ac10cf25238f93af68e/download), 2011.

[10]. P. G. Manish and S. S. Salankar. "Time series forecasting of packet loss rate using artificial neural network based on particle swarm optimization." International Journal of 
Engineering Research and General Science, 3(2): 466-472, 2015.

[11]. R. J. Manish and H. H. Theyazn. "A review of network traffic analysis and prediction techniques," pp. 1-22. Retrieved on 28 May 2020 from https://arxiv.org/abs/1507.05722, 2015.

[12]. Z. Ming and L. Yanhong. "Adaptive network traffic prediction algorithm based on BP neural network." International Journal of Future Generation Communication and Networking, 8(5):195-206, 2015.

[13]. M. M. Mohamed M. Kiarash and R. Mehdi. "Modeling of self-similar network traffic using artificial neural networks." 4th International Conference on Computer and Knowledge Engineering. Acceesed on 2 July 2020 from https://ieeexplore.ieee.org/xpl/conhome/697475 3/proceeding, 2014.

[14]. N. Piedra, J. Chicaiza, J. López and J. García. "Study of the application of neural networks in internet traffic engineering." International Book Series "Information Science and Computing", Advanced Research in Artificial Intelligence: 33 - 47, 2014.

[15]. G. Rutka. "Neural network models for internet traffic prediction." Electronic and Electrical Engineering - Kaunas Technologija, 4(68): 55 58, 2006.

[16]. G. Rutka. "Network traffic prediction using ARIMA and neural networks models." Electronic and Electrical Engineering - Kaunas Technologija, 4(84): 47 - 52, 2008.

[17]. K. G. Sheela and S. N. Deepa. Review on methods to fix number of hidden neurons in neural networks. Mathematical Problems in Engineering. Vol. 2013, Article ID 425740, 11 pages. Hindawi Publishing Corporation. http://dx.doi.org/10.1155/2013 /425740., 2013.

[18]. H. Song and L. Gan. "The research on the prediction of the network traffic based on the improved IAC-GRAY method" CHEMICAL
ENGINEERING TRANSACTIONS, 46: 1297 132, 2015.

[19]. G. Sun. "Network traffic prediction based on the wavelet analysis and hopfield neural network." International Journal of Future Computer and Communication, 2(2), 2013.

[20]. M. F. Zhani and H. Elbiaze. Analysis and prediction of real network traffic. Journal of networks. 4(9): 855 - 865, 2009.

[21]. M. Czarkowski, S. Kaczmarek and M. Wolff. "Influence of Self-Similar Traffic Type on Performance of QoS Routing Algorithms." Intl. Journal of Electronics and Telecommunications, VOL. 62, NO. 1, PP. 81-87, 2016.

[22]. M. Pagano. "Self-similarity and Long Range Dependence in teletraffic." Accesssed June 2020 from https://ieeexplore.ieee.org/xpl/ conhome /8873525 / proceeding, 2019.

[23]. Z. Maharramov, V. Abdullahez and T. Mamadova. "Modelling self-similarity traffic of multiservice network. Eureka, Physics and Engineering. Accessed on June 2020 fromhttp://eu-jr.eu/engineering /article/view/841/836, 2019.

[24]. S. A. Isaakidis, I. N. Dimou, T. D. Xenod and N. A. Dris. "An artificial neural network predictor for tropospheric surface duct phenomena." Nonlinear Processes in Geophysics, 14: 569 573, 2007.

[25]. V. M. Volkkarane and Jue J. P. "Prioritized burst segmentation and composite burst-assembly techniques for QoS support in optical burstswitched networks." IEEE journal on Selected Areas in Communications 21 (7), 1198-1209, 2002.

[26]. P. Vikas. "Self-similar traffic modeling in computer communication networks." Accessed June 2020 from https:// pdfs.semanticscholar.org/d4d3/23bae9a0646e4d 943b1d512ae4bab24838f0.pdf, 2003. 


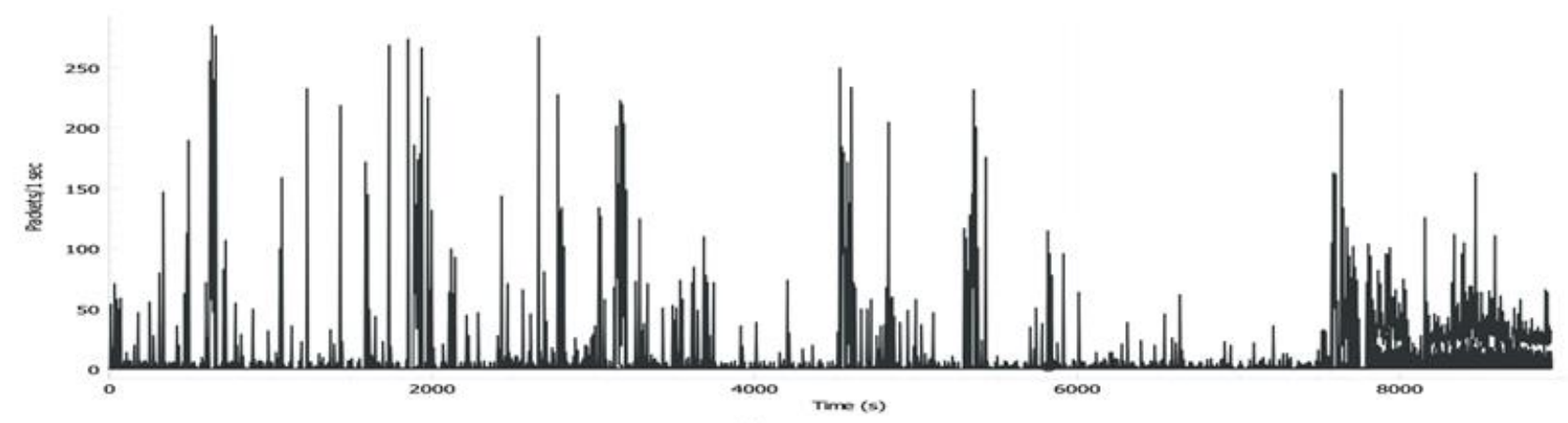

(a)

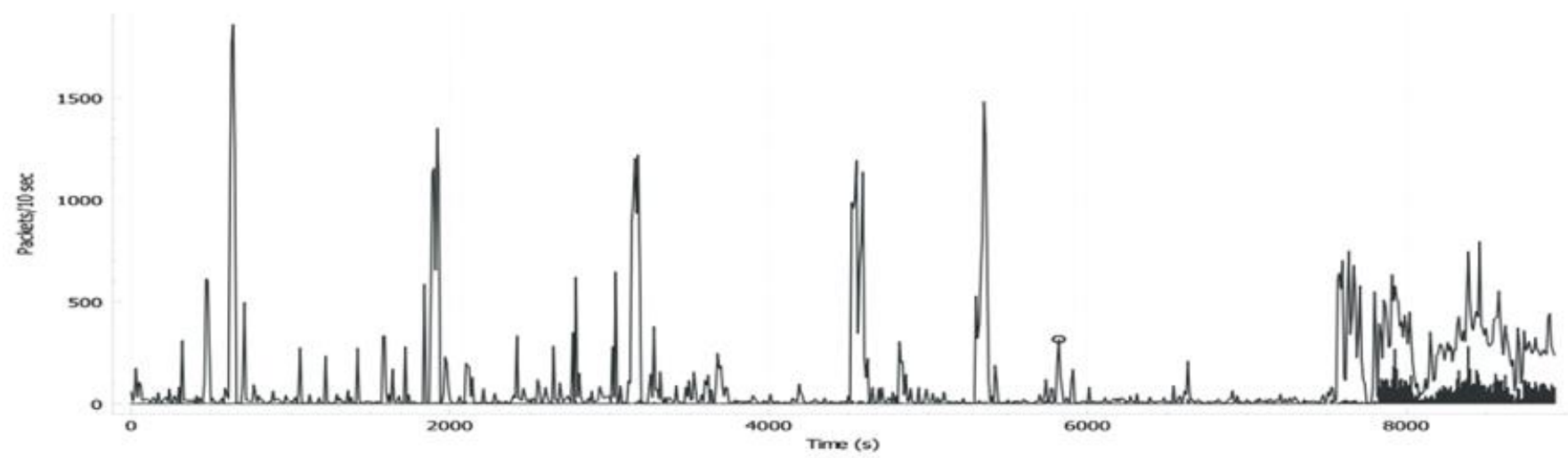

(b)

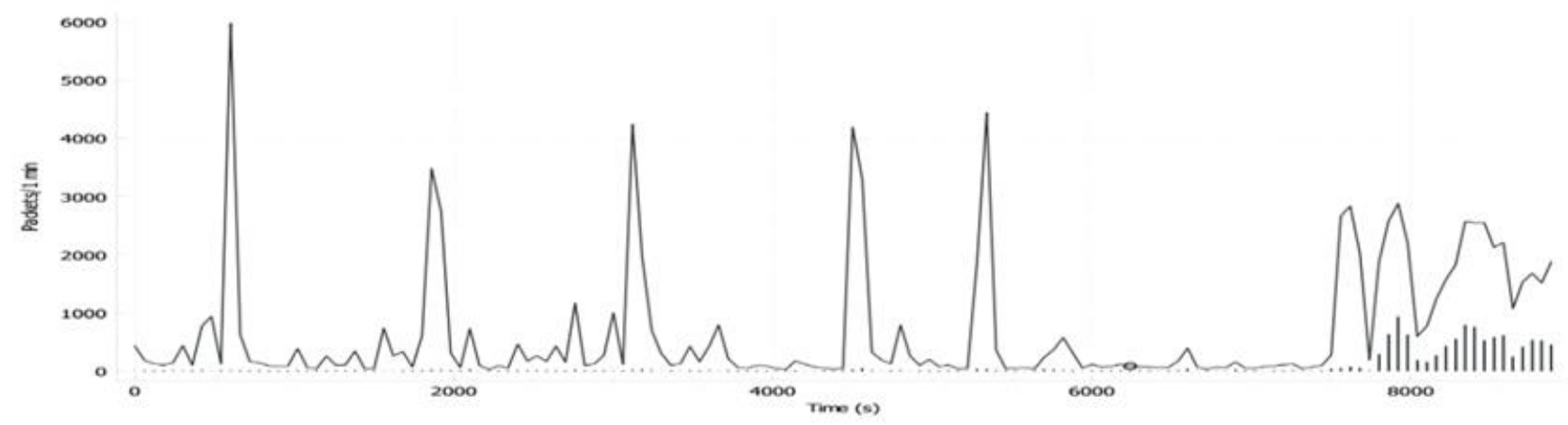

(c)

Figure 2 : Measured Test traffic for FCE - DataCap8 Captured by Wireshark 


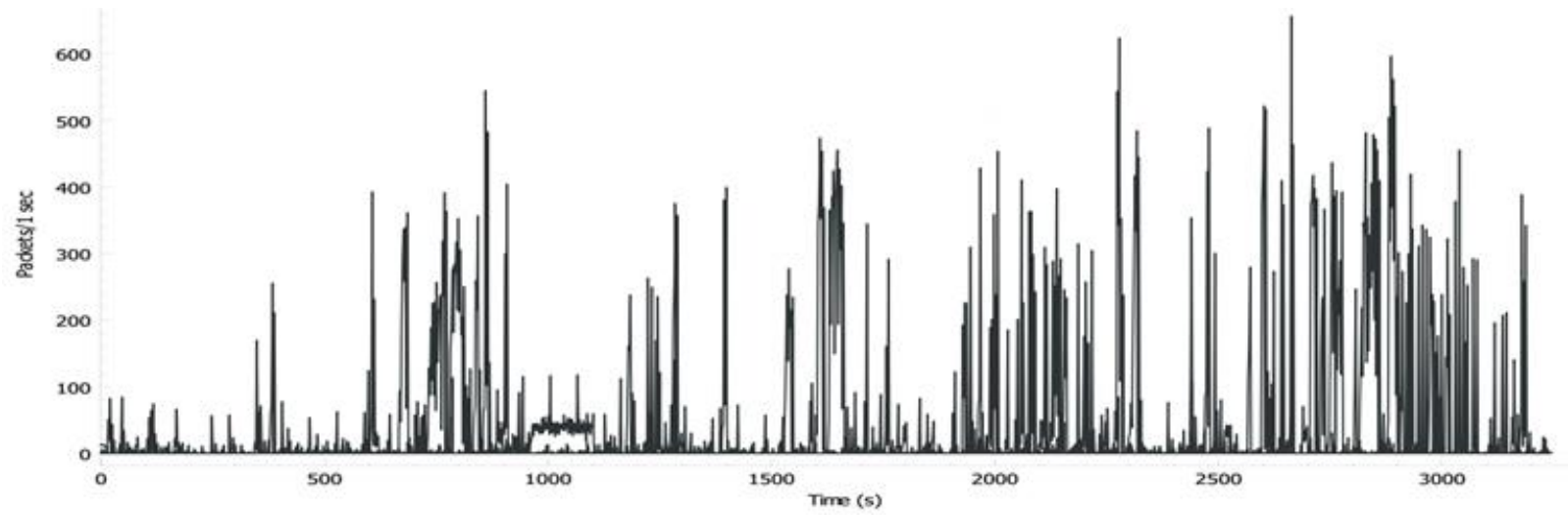

(a)

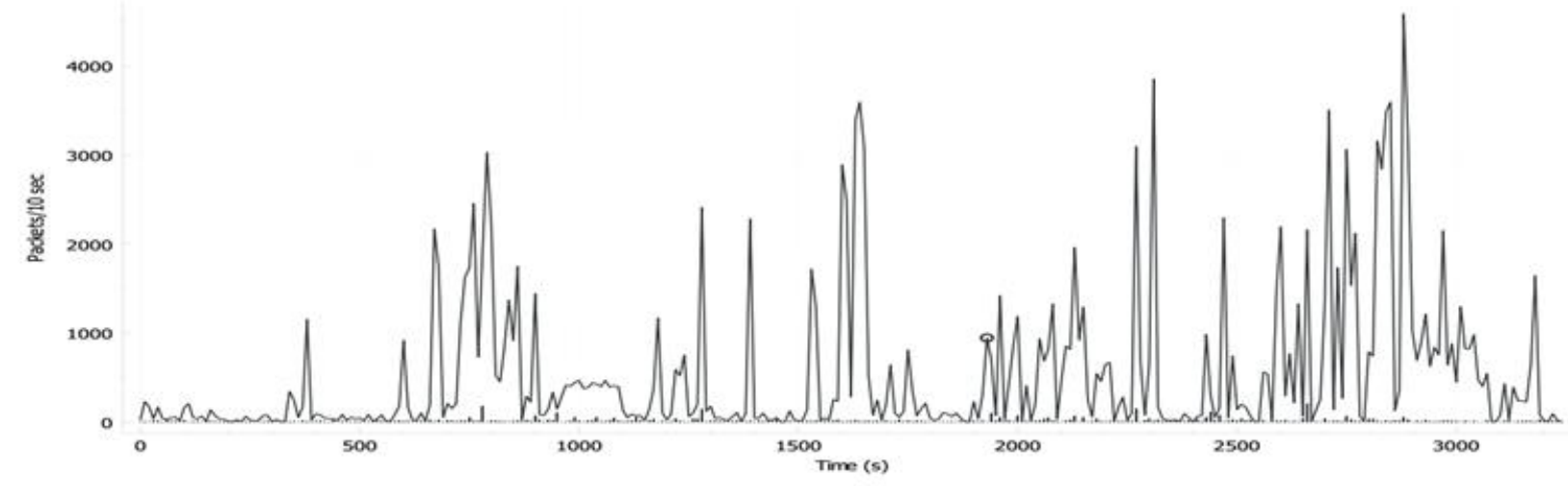

(b)

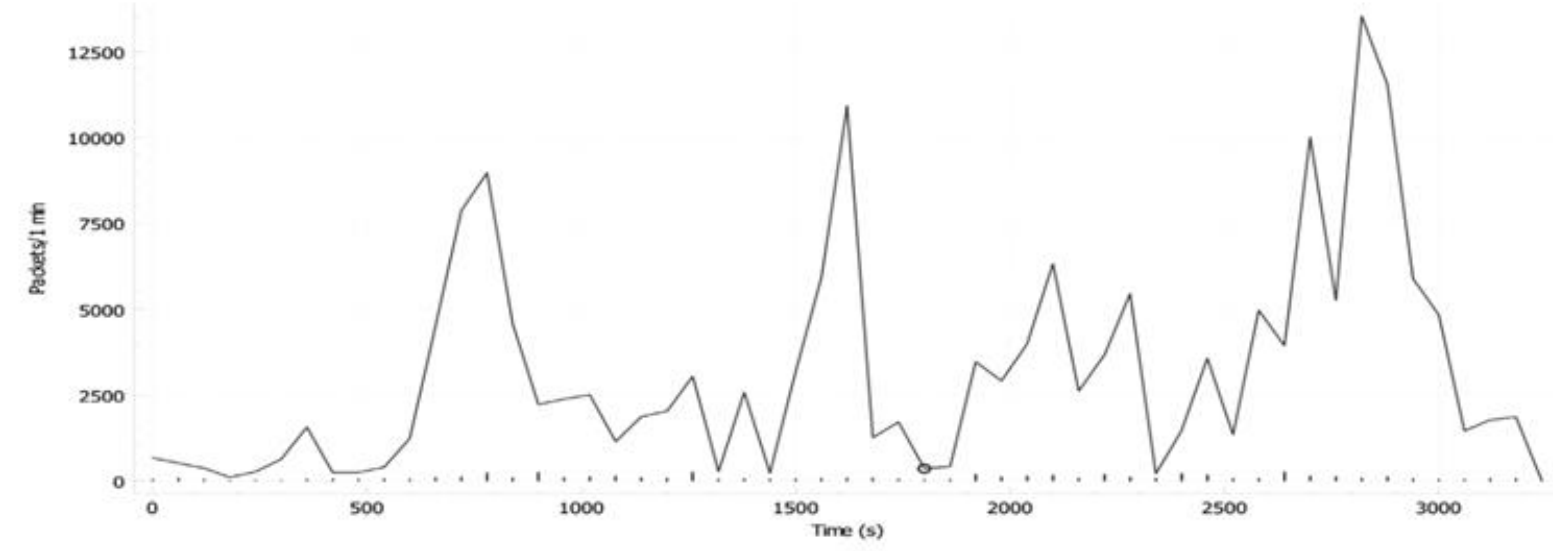

(c)

Figure 3: Measured Test traffic for MTN - DataCapMTN Captured by Wireshark

\section{Cite this article as :}

Ikharo A. B., Anyachebelu K. T., Blamah N. V., Abanihi V. K., "Optimising Self-Similarity Network Traffic for Better Performance", International Journal of Scientific Research in Science and Technology (IJSRST), Online ISSN : 2395-602X, Print ISSN : 2395-6011, Volume 7 Issue 4, pp. 164-176, July-August 2020. Available at doi : https://doi.org/10.32628/IJSRST207413

Journal URL : http://ijsrst.com/IJSRST207413 